\title{
Bioanalytical techniques in discrimination between therapeutic and abusive use of drugs in sport.
}

\begin{abstract}
The discrimination between therapeutic and abusive use of drugs in sports is performed using threshold concentrations or reporting levels, and the detection of the substances in a sample is only reported as an adverse analytical finding when the concentration exceeds the threshold or the reporting level. In this paper, the strategies of discrimination and the analytical methods used for the main groups of substances where the distinction is needed (beta-2-agonists, ephedrines, glucocorticoids and morphine) will be reviewed. Nowadays, liquid chromatography coupled to mass spectrometry is the method of choice for the analysis of these substances and, in most of the cases, a simple dilution of the urine sample is performed before the chromatographic analysis.
\end{abstract}

\section{Introduction}

The use of drugs to improve the performance in sports is banned. There is a list of groups of substances and methods forbidden in sports, which is updated every year by the World Antidoping Agency (WADA) [1]. The criteria to incorporate a substance or a method in the prohibited list are: i) potential to enhance or enhances sport performance; ii) represents an actual or a potential health risk to the athlete; and iii) violates the spirit of sport. At least two of these criteria shall be met for inclusion in the list. In addition, a substance or a method with the potential to mask the use of other prohibited substances or prohibited methods can be also forbidden. Based on these criteria, the prohibited list includes several groups of substances and methods prohibited at all times (in- and out-of-competition, IC and OOC, respectively) (non-approved substances; anabolic agents; peptide hormones, growth factors, related substances and mimetics; beta-2-agonists; hormone and metabolic modulators; diuretics and masking agents;manipulation of blood and blood components; chemical and physical manipulation; gene doping), substances prohibited only IC (stimulants; narcotics; cannabinoids; glucocorticoids) and substances prohibited in particular sports (alcohol; beta-blockers) [1].

In principle, all drugs prohibited in sports are allowed for therapeutic use, if applicable, provided that there is a proven medical need and the athlete has been granted a therapeutic use exemption (TUE). Among the forbidden drugs, ergogenic effect or serious health risks of some of them have only been demonstrated when they are administered at very high doses (supra therapeutic doses) or using certain administration routes. Because these substances are also widely used in therapeutics to treat diseases common in athletes, their use is allowed for therapeutic purposes (e.g. some beta-2-agonists and glucocorticoids). Therefore, a criterion to discriminate between therapeutic use and abuse is needed. Other drugs, such as ephedrines 
(within the group of stimulants), which are allowed OOC and prohibited IC, a discrimination criteria is required to differentiate their administration for therapeutic purposes before the competition period. For IC use, a TUE is required for ephedrines. In order to protect athletes that need to be treated with these drugs, good discrimination criteria have to be developed and this is not an easy task because the ideal criterion should be able to produce no false positive results, to protect innocent athletes, and to produce the lower number possible of false negative results, to be effective in detecting cheating athletes.

The first step for an efficient discrimination is to clearly define what is considered therapeutic use and what is considered abuse for each type of drugs, in other words, what is allowed and what is forbidden. . For beta-2-agonists, the compounds, the administration routes and the maximum doses allowed have been included in the lasts editions of the prohibited list. For glucocorticoids, that are prohibited IC only, the prohibited list describes the administration routes that are forbidden. However, as it will be described in next sections some of the allowed administration routes produce similar effects, at least for some of the compounds, than forbidden routes and, therefore, additional revision of the criteria needs to be performed.

The second step is to define the discrimination strategy between allowed and forbidden administrations. The strategy followed by WADA for most of the compounds is to define threshold concentrations for the parent drugs. Threshold concentrations based on pharmacokinetic studies with evaluation of the urinary concentrations of the drug or its metabolites after administration of the maximum allowed doses in a representative population of volunteers would be advisable. The current threshold concentrations for most of the compounds have been established based on drug administration studies (e.g. salbutamol, pseudoephedrine) or on clinical data from drug manufacturing companies (e.g. formoterol). However, this data is not available for all the compounds and this is the reason why the criteria have been changed over time for some of the compounds as soon as new information has been obtained.

Table 1 describes, among others, the threshold concentrations defined for salbutamol and formoterol (beta-2-agonists), for ephedrines and for morphine [2]. Apart from the drugs where distinction between therapeutic use and abuse is needed, the list of threshold substances also includes compounds of endogenous nature (19norandrosterone, glycerol) for which distinction between endogenous production and exogenous administration is needed, and the metabolite of tetrahydrocannabinol for which a threshold concentration is used to distinguish recent use from the long residual presence of the metabolite for several days after an authorized used (cannabis is only forbidden in samples collected in competition). 19-Norandrosterone can be also result of the permitted use of contraceptives containing norethisterone; in this case, the additional detection of specific metabolites of noretistherone is used for discrimination.

According to WADA rules, the detection of a threshold substance in a sample is only reported as an adverse analytical finding (AAF) when the concentration exceeds, with an appropriate level of confidence, the threshold concentration. The confidence interval is established taking into account a maximum measurement uncertainty defined for the quantitation of each threshold substance. Based on the threshold concentration and 
the confidence interval, a decision limit has been defined for each one of the substances (Table 1). The decision limit is the concentration above which it can be decided that the threshold concentration has been exceeded with a statistical confidence of at least $95 \%$ [2]. For threshold substances, a quantitative method has to be applied for confirmation purposes to verify that the concentration of the samples is greater than the decision limit. Quantitative methods have to comply with specific requirements for validation and with the criteria of maximum combined standard uncertainty defined for each threshold substance. For salbutamol, formoterol and ephedrines, if they are detected at concentrations below the decision limits in conjunction with a diuretic or other masking agent, both substances shall be confirmed and reported as AAF. In this case, the confirmation of the threshold substance requires only the identification of the compound and not its quantitation.

For glucocorticoids the strategy followed by WADA is different. Glucocorticoids are only forbidden IC when used by systemic routes (oral, intravenous, intramuscular (IM), and rectal) and allowed for therapeutic use using other administration routes, and the current criterion of discrimination between allowed and forbidden use is not based on a threshold concentration. In this case, probably due to the wide number of compounds within the group of glucocorticoids, and the different administration ways and doses, and the availability of few pharmacokinetic data, in an attempt to make the distinction between use and abuse, WADA defined a general reporting level of $30 \mathrm{ng} / \mathrm{mL}$ for all glucocorticoids and their metabolites, and laboratories are not to report below this concentration [3]. In the case of glucocorticoids, a quantitative method is not required and the decision whether the concentration is below or above the reporting level is taken based on the comparison of the sample from the athlete with a quality control sample spiked with the compound at the reporting level.

In recent years, an important research activity has been developed in this area with the aim to verify the current discrimination criteria and to improve the criteria when necessary. The research has been focused in two main aspects: metabolic and pharmacokinetic studies to obtain data on urinary concentrations of the compounds and their metabolites after different doses and/or administration routes, and optimization of the analytical methods to improve the quality of analytical results. The objective of this paper is to review the pharmacokinetic/metabolic data available and the bioanalytical techniques used to discriminate between therapeutic and abusive use of drugs in sports. The paper will be mainly focused on the main groups of substances where the discrimination is needed: beta-2-agonists, ephedrines, glucocorticoids and morphine. 


\section{Beta-2-agonists}

Agonists of the $\beta_{2}$-adrenergic receptors (beta-2-agonists) are prohibited in sports because they have central nervous system stimulation activity and, also, because some of them (e.g. salbutamol) have some anabolic activity when administered at high doses or using systemic administrations [4]. Their status in the WADA Prohibited List has changed substantially along the years, involving some time the need for requesting a previous notification or the need of a TUE. Reports on the use of beta-2-agonists at the Olympic Games may be found elsewhere [5,6]. In the year 2000, the Medical Commission of the International Olympic Committee (IOC) established a selected list of potential usable agents, and for salbutamol the consideration of an AAF only if the concentration of free plus glucuronide salbutamol exceeded the threshold of $1 \mu \mathrm{g} / \mathrm{mL}$. Nowadays, the regulation of the 2015 WADA Prohibited List indicates that all beta-2agonists are prohibited except inhaled salbutamol (maximum treatment of $1600 \mu \mathrm{g}$ over 24 hours), inhaled formoterol (maximum delivered dose of $54 \mu \mathrm{g}$ over 24 hours) and inhaled salmeterol (in accordance with the manufacturers' recommended dose).

The previous rule suggests the need for establishing some decision limits on the urinary concentrations of these compounds to consider a finding for an AAF. Threshold concentrations have been established for salbutamol and formoterol (Table 1). The threshold for salbutamol is based mainly on the pioneering work by Ventura et al [7] where it was shown that the administration of the maximum inhaled dose gives rise usually to concentrations of about $500 \mathrm{ng} / \mathrm{ml}$, and a margin of variability of twice this value was established by the IOC Medical Commission. For formoterol, the threshold arises from clinical data from the drug manufacturing company and more recent administration studies [8,9]. In fact, according to the 2015 WADA Prohibited List, the presence in urine of salbutamol in excess of $1 \mu \mathrm{g} / \mathrm{mL}$ or formoterol in excess of 40 $\mathrm{ng} / \mathrm{mL}$ is presumed not to be an intended therapeutic use unless the athlete proves, through a controlled pharmacokinetic study, that the abnormal result was the consequence of the use of a therapeutic inhaled dose up to the maximum indicated above. In real analytical practice, and taking into account the maximum combined standard uncertainty allowed (Table 1) the established decision limits are respectively $1200 \mathrm{ng} / \mathrm{mL}$ for salbutamol and $50 \mathrm{ng} / \mathrm{mL}$ for formoterol [2]. Some studies suggest that these thresholds are rather conservative $[9,10]$ thus allowing some false negative results, while other reports indicate that in rare cases the threshold might be exceeded after therapeutic use, which would involve false positive results [11]. The wide interindividual variability and urine dilution may be responsible for some of these uncommon situations [12]. The threshold, however, seems appropriate for most of subjects [13], especially if corrected for dilution by the specific gravity of the urine [14].

The analysis of beta-2-agonists for doping control has been historically accomplished through derivatization of polar functional groups and subsequent analysis by gas chromatography coupled to mass spectrometry (GC-MS) $[7,15,16]$. The procedures involved an initial enzymatic hydrolysis of the urine with $\beta$-glucuronidase. Then, the free and hydrolized compounds were extracted using liquid-liquid or solid-phase extraction and the dried extracts were derivatized with $\mathrm{N}$-methyl-N-trimethylsilyl-trifluoroacetamide or a mixture based on that reagent (e.g. MSTFA:NH 4 l:2-mercaptoethanol 1000:2:6) $[7,16]$. Other chemical agents were also useful for derivatization, and a summary of the main ones can be found elsewhere [15]. Of particular interest for the additional 
evidences afforded for identification is the two-steps derivatization to form cyclic methylboronates (MB) in the first step followed by formation of trimethylsilyl (TMS) derivatives in the second step [17]. After each derivatization step, GC-MS analysis was performed obtaining salbutamol-di-MB, formoterol di-MB and salmeterol di-MB in the first step, and salbutamol tris-O-TMS, formoterol bis-O-TMS, and salmeterol tris-OTMS, respectively, after the second step. This procedure yields complementary mass spectrometry diagnostic data affording information for unambiguous identification.

The huge developments of the combination between liquid chromatography and mass spectrometry (LC-MS) in recent years have driven the shifting of the analysis of beta-2agonists toward such a technology. Initially, the shift was done only on the separation methodology, but maintaining the sample preparation (hydrolysis and extraction) similar to the one used for GC-MS methods. In this regards, limits of detection of salbutamol of about $10 \mathrm{ng} / \mathrm{ml}$ urine were achieved [18]. However, because the need for sensitivity was not a limiting factor in doping control given the high decision limit established, a more practical approach involving a direct injection of $1: 1$ diluted urine into the LC-MS system was also developed, especially for confirmation analysis [19]. Suitable isotopically labeled internal standards were used for quantitative confirmation analysis. A triple quadrupole mass spectrometer with an electrospray source working in positive ionization mode and multiple reaction monitoring acquisition mode, coupled to chromatographic columns packed with small particles allowed total running times of 3.2 minutes per sample, thus giving analysis times per batch (calibration samples, quality control samples and replicates of the sample) lower than one hour [19]. Slight better sensitivity was accomplished with a more concentrated dilution $(4: 1)$ with acetonitrile [20]. These methods did not attempted to quantify salbutamol glucuronide, but subsequent work [21] indicated that this phase II metabolite accounts only to less than $3 \%$ of the unchanged salbutamol. Instead, the analysis of the phase II metabolite sulfated salbutamol [20] may be interesting as alternative marker of orally administered salbutamol however the threshold concentration would need to be reevaluated using that marker. Some increase in sensitivity may be accomplished with other instrumental configurations (e.g. quadrupole/orbitrap mass spectrometer), thus allowing to quantify salbutamol in other matrices such as dried blood spots [22]. The use of LC-MS for targeted and non targeted metabolomics may be interesting in the near future for beta2-agonists and other agents [23].

The use of chiral chromatography to separate enantiomers of salbutamol demonstrated to be useful to discriminate between therapeutic inhaled use and abusive oral use of salbutamol [24]. Salbutamol has a single asymmetric carbon atom, and it is administered as a racemic mixture. Disposition studies after administration of the drug by different routes demonstrated that the enantiomers are conjugated at a different rate by the body tissues. After oral intake of salbutamol, the unchanged $\mathrm{S}(+)$ enantiomer is excreted in a greater proportion than the $\mathrm{R}(-)$ isomer. However, the enantiomeric ratio after inhalation is close to unity. Therefore, the proportion of metabolites corresponding to both enantiomers will differ depending on the route of administration. The feasibility of such an approach for the benefit of doping control was established by Bergés et al [24,25], using a quiral column of (S)-indoline-2-carboxylic acid linked to (R)-1-(alphanaphtyl)ethylamine urea. As the proposed methodology [25] was based on fluorometric detection (excitation and emission wavelengths of 230 and $309 \mathrm{~nm}$, respectively), urine 
samples had to be purified for a clean background, with solid phase extraction yielding the best results. The outcome indicated that the simultaneous evaluation of absolute concentration in urine of salbutamol and the ratio between the enantiomers are a useful tool to differentiate prohibited oral and permitted inhaled administrations.

The current methods used for the quantitation of formoterol involve LC-MS/MS analysis using isotopically labelled formoterol as internal standard and previous enzymatic hydrolysis and solid-phase extraction of the urine sample [8]. LC-MS/MS system used a classical water-acetonitrile mobile phases containing ammonium formate. Positive electrospray ionization mode was used to reach a limit of detection of $0.1 \mathrm{ng} / \mathrm{mL}$. Similar approaches involving subtle methodological changes have been proposed by other authors [26]. Sample preparations consisting only in enzymatic hydrolysis and dilution of the urine sample [27,28] or liquid-liquid extraction with ethyl acetate [29] have been also described. Results of the quantitative analysis of formoterol in samples subjected to enzymatic hydrolysis and dilution before LC-MS/MS analysis are shown in Figure 1. Some of the studies described in the literature include the detection and quantitation of the O-demethylated metabolite of formoterol [27].

245 For salmeterol, there is not any threshold proposed for the distinction between permitted and prohibited use, but analytical methodology exist for the quantitation of salmeterol in urine. Methods mentioned above by GC-MS $[15,17]$ are suitable, but there are also new developments based on LC-MS/MS [30], able to detect salmeterol in urine up to 48 hours after an inhaled application of $100 \mu \mathrm{g}$. In any case, the urinary concentrations to target are relatively low (maximum $1.3 \mathrm{ng} / \mathrm{mL}$ ) given the low dose to be administered and the low amount of drug reported excreted unchanged $(0.27 \%$ in first 12 hours) [30]. 
256 Ephedrines are a class of sympathomimetic amines comprising norephedrine 257 (phenylpropanolamine, NEPH), norpseudoephedrine (cathine, CAT), ephedrine (EPH), 258 pseudoephedrine (PSE) and methylephedrine (MET), and they are one of the oldest 259 groups of therapeutically used stimulants. Some of the ephedrines are commonly found 260 in over-the counter (OTC) preparations to treat cough, cold and allergy. They can also 261 be found in dietary supplements [31,32] and products containing ephedra-derived alkaloids [33,34]. Their administration can have positive effects on sports performance because of their activity on the respiratory or cardiovascular system.

Ephedrines are in the list of prohibited substances in sports since long time ago to control their use. They are prohibited IC. Due to the frequent therapeutic administration and the debated ergogenic effect, the regulations are difficult and threshold concentrations were established. Until 2003, threshold concentrations were: for CAT 5 $\mu \mathrm{g} / \mathrm{ml}$, for EPH and MET $10 \mu \mathrm{g} / \mathrm{ml}$, for NEPH $10 \mu \mathrm{g} / \mathrm{ml}$, and for PSE $25 \mu \mathrm{g} / \mathrm{ml}$. In 2003, $\mathrm{NEPH}$ was removed from the list due to the lack of evidences of ergogenic effect even at high doses. On the other hand, for positive cases declared for PSE using the threshold concentration of $25 \mu \mathrm{g} / \mathrm{ml}$, the athletes often claimed that they were due to inadvertent administration of OTC preparations. Studies demonstrated that concentrations in urine greater than the threshold $(25 \mu \mathrm{g} / \mathrm{ml})$ could be obtained for some individuals after therapeutic administration of the compound. After these results, PSE was removed from the prohibited list in 2003 and included in the monitoring program in order to detect patterns of misuse in sports. After several years in the monitoring program, an increase in the number of samples with high urinary concentrations of PSE (greater than $75 \mu \mathrm{g} / \mathrm{ml}$ ) was detected [35]. In addition, a clear evidence of PSE abuse in specific sports and regions of the world was observed [36]. Moreover, during that period, the evaluation of anti-doping results of CAT (with threshold concentration of $5 \mu \mathrm{g} / \mathrm{ml}$ and metabolite of PSE) was problematic in the presence of PSE, a permitted drug. For these reasons, PSE was reintroduced in the WADA prohibited list in 2010, and existing and additional experimental data [35-38], let to the definition of a new threshold concentration for PSE at $150 \mu \mathrm{g} / \mathrm{ml}$ (Table 1). Due to the influence of diuretics on the excretion of ephedrines [39], it is also established in the WADA rules that if an ephedrine is detected in a sample at levels below the decision limit in conjunction with a diuretic, both substances have to be reported as AAF [2].

For ephedrines, the difficulty of the analysis lies on the need of separation of the diastereoisomeric pairs (NEPH and CAT, and EPH and PSE). The pairs have the same elemental composition and, consequently, they share the same mass spectra and require chromatographic separation for unambiguous identification and accurate quantitation. The former methods to analyze these compounds were based on a liquidliquid extraction followed by gas chromatography with nitrogen phosphorus detection (GC-NPD) or GC-MS [40-42], or by high performance liquid chromatography with ultraviolet detection (HPLC-UV) [43,44]. In both cases, the use of very slow gradients or isocratic conditions was required to obtain the adequate resolution between the diastereoisomeric pairs. Separations of the diastereoisomeric pairs by GC-MS using complex derivatization procedures were also described [45]. 
In these former methods, a sample preparation procedure consisting of a liquid-liquid extraction with diethyl ether or tert-butylmethyl ether at $\mathrm{pH} 14$ was used [40-43]. In most of the cases, the organic extract was directly injected into the chromatograph. If evaporation of the organic solvent was needed to pre-concentrate the sample before the chromatographic analysis, soft evaporation conditions (nitrogen pressure, temperature of the water bath) had to be used in order to avoid losses of the analytes during the evaporation step. A method to quantify ephedrines in urine samples without

The introduction of LC-MS offered a good alternative for the analysis of ephedrines [20,46-52]. Ephedrines have a nitrogen atom and have a very high ionization efficiency using electrospray ion source, resulting in very high sensitivity. The high sensitivity for ephedrines by LC-MS and the compatibility of the system with the aqueous matrix of the urine permits the reduction of the sample preparation process, and direct dilution and injection of the urine into the LC-MS systems is currently the most widely used strategy among antidoping laboratories to detect and quantify ephedrines in urine $[20,46,47,49-52]$. In general, the separation of the diastereomeric pairs is performed during the confirmation procedure, after detection of the presence of ephedrines in a sample during the initial testing procedure.

In most of the works published in literature, separation of ephedrines is accomplished using reversed phase liquid chromatographic columns with acidic mobile phases. In most cases C18 columns were used [20,48,52], however ephedrines are high hydrophilic compounds and they show poor retention in reversed phase columns, and in some works used C8 columns to increase retention [46,47]. In these conditions, baseline separation of the diastereomeric pairs is achieved using isocratic elution 0 very slow gradient elution with mobile phases with high aqueous content and very low content or organic modifier. An example of the separation of ephedrines using C18 column with a very slow gradient elution using water and acetonitrile with $0.1 \%$ of formic acid is shown in Figure 2 [52]. Gray et al [49] described the use of a high pH mobile phase in order to suppress analyte ionization and thus alter their polarity resulting in enhanced retention. In these conditions, $\mathrm{APCl}$ yielded greater stability and reproducibility of the signal compared to ESI. Another strategy is the use of hydrophilic interaction liquid chromatography (HILIC) [50,51]. Compared to C18 reversed-phase chromatography using high $\mathrm{pH}$ mobile phases, HILIC showed benefits in terms of peak shape, sample loading capacity and increased sensitivity by ESI. Common problems associated with HILIC including retention shifts and undesirable peak shapes were solved using adequate re-equilibration times and injection solvents [51].

Analysis of ephedrines has been performed using different mass spectrometric analyzers. Former LC-MS methods used ion-trap mass spectrometry and MS/MS analysis was used for quantitative purposes [46]. Nowadays, although most of the authors propose the use of tandem mass spectrometry using triple quadrupole instruments $[20,47]$ Q-TOF instruments have also shown reliable quantitative results $[48,51]$. 


\section{Glucocorticoids}

350 Glucocorticoids are synthetic analogs of cortisol, used as anti-inflammatory and 351 immunosuppressive agents since 1950s [53]. Glucocorticoids can be administered by 352 different routes for the treatment of different pathologies, and they are also widely used in sports to treat conditions such as asthma and acute injuries. Due to some evidences of performance enhancing effects and the important health risks associated with their use in therapeutics, because of the inhibition of the hypothalamic-pituitary-adrenal axis, the use of glucocorticoids in sports is controlled [54]. Since their inclusion in the prohibited list in 1986, there has been a great controversy regarding the glucocorticoids status in the prohibited list because the main reason to be forbidden seems to be the health risks associated with glucocorticoids therapy. The compromise found was to ban glucocorticoids only IC and only when administered by the so-called systemic routes (oral, intravenous, IM and rectal administrations). Until 2010, a declaration of use of the athlete was required to use glucocorticoids by intraarticular (IA), periarticular, peritendinous, epidural, intradermal and inhalation routes, and topical administrations were not prohibited and neither required TUE nor a declaration of use. Nowadays, all these other administration routes, considered of local action, are allowed for therapeutic use without TUE or declaration of use.

In an attempt to distinguish forbidden and allowed administrations, WADA defined a general criterion for all glucocorticoids at $30 \mathrm{ng} / \mathrm{mL}$ and laboratories are not to report as AAF samples with glucocorticoids below this concentration [3]. That criterion is based on general considerations and not in pharmacokinetic/metabolic studies of the different glucocorticoids after different administration routes, and it has shown to be not suitable, at least, for some of the compounds.

In consequence, a great research activity has been developed in recent years to help in the definition of a better criteria of discrimination between allowed and forbidden administration routes [55-69]. The metabolism of several glucocorticoids has been revisited using LC-MS/MS analysis (budesonide, betamethasone, methylprednisolone, prednisolone, prednisone and triamcinolone acetonide) [59-61,64,66,68]. LC-MS/MS has demonstrated to be very useful for the identification of new glucocorticoid metabolites compared to the limitations of GC-MS analysis, due to the difficulties to obtain adequate derivatives of polyoxigenated metabolites to make them amenable to GC-MS analysis. Moreover, the characteristic ionization and collision induced dissociation (CID) pattern observed for glucocorticoids using electrospray ionization was the basis to create a series of open scan methods (neutral loss and precursor ion scan methods) to detect new glucocorticoid metabolites in samples obtained after administration of the compounds. Another strategy used to detect new metabolites was the use of selected reaction monitoring methods that included theoretical ion transitions of potential metabolites considering hydroxylations, oxidations and reductions, based on the characteristic CID behavior. Using these LC-MS/MS strategies a high number of metabolites, not previously reported, were detected for most of the studied compounds resulting from oxidations, reductions, hydroxylations or cleavages of the side chains $[60,61,64,66,68]$. The structure of some of the metabolites detected was demonstrated using synthetized standards of the compounds. For other metabolites, the structure 
was proposed based on mass spectrometric data and comparison of the data with the synthetized compounds needs to be performed before definitive structure assignment. As an example, the metabolites detected after oral administration of prednisolone are described in Figure 3 [68].

Clinical studies were performed consisting of the administration of glucocorticoids to healthy volunteers using allowed and forbidden routes. Concentrations of the parent drugs and the metabolites were measured in urine using LC-MS/MS analysis, to look for the best marker of discrimination for each glucocorticoid [62,63,65,67]. As new data is available, it seems almost impossible to have a general criterion for all glucocorticoids for several reasons: the high number of compounds, the diversity of administration routes, the differences in the metabolic patterns and the different pharmacological activity and, thus, the different therapeutic doses, among others.

Results obtained up to now have been used to improve the discrimination criteria for some glucocorticoids. An example is budesonide, the glucocorticoid with the highest number of AAF in the lasts years [70]. Metabolic studies performed using LC-MS/MS $[60,62]$, indicated that the best marker to discriminate between inhaled therapeutic use and forbidden oral administration is the metabolite $6 \beta$-hydroxy-budesonide. That metabolite drastically reduces the number of false positive results after inhalation compared with the main budesonide metabolite, 16a-hydroxy-prednisolone, proposed by previous authors $[71,72]$. Figure 4 shows chromatograms of urine samples collected before administration and after inhaled and oral administrations of budesonide [60]. 6 $\beta$ hydroxy-budesonide has been recently adopted in WADA regulations as a marker for budesonide [3].

Regarding other glucocorticoids, in general, samples collected after permitted administration routes (topical, intranasal, inhaled) have concentrations of the parent compounds lower than the current WADA criterion at $30 \mathrm{ng} / \mathrm{ml}[56,57,63-65,67]$. Usually, after oral administrations samples showed concentrations greater than 30 $\mathrm{ng} / \mathrm{mL}$, at least for a period of time $[55,56,63,65]$. However, this criterion is not sensitive enough to detect low IM administrations of some glucocorticoids such as triamcinolone acetonide [64,67], although it has been shown to be suitable to detect high IM doses [69]. For some glucocorticoids, the use of specific metabolites instead of the parent drug would improve the discrimination between administration routes [62,63]. As an example, for methylprednisolone metabolites resulting from oxidation of the hydroxyl group in position 11, and subsequent hydroxylation in position 16 or reduction in position 20 are the best markers to differentiate between topical and oral administrations [62]. Additional studies are needed to define more suitable markers compared with the current criteria.

However, one of the main drawbacks of the current criterion of discrimination is that samples collected after allowed IA or periarticular administrations can produce concentrations in urine greater than $30 \mathrm{ng} / \mathrm{mL}[55,65,69]$. In addition, the metabolic profile of metabolites of several glucocorticoids (betamethasone, triamcinolone acetonide, methylprednisolone) after IA or periarticular administration is similar to IM administration and these results indicate the difficulty to distinguish these routes of administration $[59,65,69]$. Moreover, it has been demonstrated that IA administration, and probably periarticular administration, of these glucocorticoids (e.g. betamethasone 
or triamcinolone acetonide) produces a reduction in plasmatic cortisol concentrations and, therefore, systemic effect [73,74]. This is an additional controversial point on the criteria used by WADA: if the criteria to ban an administration pathway for glucocorticoids is the production of systemic effect, IA and periarticular administration should be banned. Therefore, a revision of the status of IA and periarticular administrations in the prohibited list needs to be performed.

Another parameter studied to distinguish systemic and non-systemic administrations is the concentration of cortisol and its endogenous metabolites. Concentrations of cortisol decrease following systemic administration but not after topical treatment [55,56,75]. However, considering the circadian rhythm of cortisol and other parameters that may affect cortisol concentrations, further studies should be carried out before the use of a criterion based on cortisol concentrations in the antidoping field.

From the analytical point of view, the sensitivity has not been the main limitation in the glucocorticoids analysis in routine doping control because of the relatively high concentrations to be measured according to WADA regulations. Even though it has been reported that glucocorticoids are excreted mainly free [76], the glucuronide fraction is important at least for some of them [60]. For this reason, an enzymatic hydrolysis with $\beta$-glucuronidase (from $E$. coli) is usually used in anti-doping laboratories $[62,63,67,72]$. Direct detection of glucuronide conjugates has also been reported [59], confirming the need to take these conjugates into consideration. After hydrolysis, if performed, samples are alkalinized (around $\mathrm{pH}$ 9) before the liquid-liquid extraction. Tert-butyl-methyl ether and diethyl ether had been chosen as organic solvents in screening methods for glucocorticoids [71,72]. However, for some polar metabolites, such as 16a-hydroxy-prednisolone, poor extraction recoveries are obtained with these solvents, thus use of a more polar solvent (ethyl acetate) is suggested $[58,62,63,67]$.

Historically, glucocorticoids were analyzed by GC-MS after derivatization of the functional groups [77]. The derivatization before GC analysis is necessary to protect functional groups and also because of instability of the side chain at $\mathrm{C17}$, which is lost usually to yield the 17-oxo-steroid. Formation of TMS derivaties or methoxyamine-TMS derivates were the most typical derivatization procedures. However, difficulties arose to obtain a single derivative because of the high number of functional groups. Moreover some structural modifications of the glucocorticoid structure could also decrease the

LC-MS/MS approaches replaced soon the GC-MS methods mainly due to the possibility to directly analyze glucocorticoids, without the need of previous derivatization, and the enhanced sensitivity obtained using electrospray ionization. Glucocorticoids can be detected in both positive and negative electrospray ionization modes [78]. They have two centers feasible for electrospray ionization: the conjugated 3-keto function, that can be protonated, and the oxygen atoms present in C17, C20 and C21 which can favor the formation of adducts (mainly with acetic or formic acid), that can be further deprotonated [78]. Moreover, some potential glucocorticoid metabolites unable to form $[\mathrm{M}+\mathrm{H}]^{+}$ion due to the lack of a conjugated ketone in the A-ring, can be detected by formation of adducts with ammonia [65,79]. The possibility of using different stationary phases combined with different mobile phases make LC more suitable to separate and identify a complex mixture of glucocorticoid metabolites. For 
example $20 \beta$-dihydro-prednisolone, one of the main metabolites of prednisolone, could not be separated from the isobaric and endogenous cortisol using methanol and water as mobile phases; however, complete chromatographic separation was achieved using acetonitrile and water as mobile phases (see retention times in Figure 3) [68]. In the initial testing procedures applied in doping control, compromised chromatographic conditions are chosen for the simultaneous analysis of several analytes, and these conditions may be not optimal for the resolution of some of them. In those cases, the selection of a selective ion transition could play a key role to discriminate the analyte from other metabolites or interferences. For example, the ion transition $\mathrm{m} / \mathrm{z} 447$ to $\mathrm{m} / \mathrm{z}$ 339 is more selective than the transition $\mathrm{m} / \mathrm{z} 447$ to $\mathrm{m} / \mathrm{z} 171$ for the detection of $6 \beta$ hydroxy-budesonide as can be seen in Figure 4 [62]. LC-MS/MS approach also allows

495 the direct detection of phase II metabolites [59]. Due to all of these capabilities of LCMS/MS technology several metabolites of glucocorticoids have been identified and studied as alternative markers to distinguish between administration routes, as explained above.

499 Besides several advantages of the LC-MS/MS systems, the most important drawback 500 is the matrix effect. The ion suppression caused by the matrix effect can compromise 501 the detection of relatively high concentrations of glucocorticoids in some samples [80]. 502 Isotopically labeled analogues of the analytes are normally used as internal standards 503 to correct for losses during the process of for the ion suppression or ion enhancement 504 effect especially when an estimation of the concentration needs to be performed $505[62,63,67,80]$. 
The use of morphine is prohibited in sport. However, a common authorized antitussive drug (codeine) is partially metabolized to morphine and this fact complicates the interpretation of results. A threshold of $1 \mu \mathrm{g} / \mathrm{mL}$ with a decision limit of $1.3 \mu \mathrm{g} / \mathrm{mL}$ 512 (maximum uncertainty of $15 \%$ ) requires quantitative analyses of any urine sample 513 containing morphine. According to the WADA rules, morphine at a urinary 514 concentration greater than the decision limit is considered an AAF unless it is 515 determined to be the result of the administration of codeine (i.e. if codeine is present in 516 the sample). Therefore, although administration of codeine might result in morphine 517 concentrations greater than $1.3 \mu \mathrm{g} / \mathrm{mL}$, the detection of codeine in the sample indicates 518 that the finding results from codeine use.

For years, GC-MS methodology has been used for the analysis of morphine in doping 521 control after $\beta$-glucuronidase hydrolysis and usually TMS derivatization [81-83]. More 522 recently, the availability of LC-MS/MS applied to hydrolyzed urine samples allowed the 523 optimization of the quantitation of morphine [20]. However, the LC-MS approach 524 opened the door to the direct quantification of morphine-3-glucuronide and unchanged 525 morphine if direct or diluted injection of the urine is carried out. In this regards, simple 526 LC-MS was used already at the Athens 2004 Olympic Games [46] and more recently, 527 hydrophilic interaction liquid chromatography high resolution/high accuracy mass 528 spectrometry has being proposed in the framework of a more general multi-targeted 529 screening anti-doping assay [84]. 
533 The discrimination between therapeutic use and abuse of drugs in sports is not an easy 534 task. The ideal criteria should be able to declare as negative, samples collected after 535 allowed administration routes and allowed doses (no false positive results), and to 536 declare as AAF, samples collected after forbidden administration pathways or 537 forbidden doses. A lot of work has been done to define the currently used criteria, 538 however some limitations still remain for some of the compounds, and research for more effective criteria is needed for these drugs.

An area of work is still to clearly define what is allowed and what is forbidden for some of the drugs. The ideal situation would be to define the maximum allowed doses and the allowed ways of administration based on pharmacokinetic/pharmacodynamic data, and the main problem is that these data are not available nowadays for all the compounds. In addition, the separation allowed/forbidden should be consistent from a pharmacodynamic point of view. In this sense, a revision of the classification of some administration routes of glucocorticoids (e.g. IA or periarticular administrations) needs to be performed. According to the prohibited list, the criterion used by WADA to forbid an administration route for glucocorticoids is based on the capacity to produce a systemic effect. IA and periarticular administrations are allowed because they are considered to have local action. However, it has been demonstrated that IA administration, and probably periarticular administration, of some glucocorticoids (e.g. betamethasone or triamcinolone acetonide) produces systemic effect. In consequence, according to these pharmacodynamic data and applying the WADA criteria of classification, IA administration should be forbidden. However, the wide use of glucocorticoids by athletes by IA or periarticular administration to treat acute injuries makes that decision difficult. One possibility to control that situation is to ask for Therapeutic Use Exemption when glucocorticoids need to be administered by IA or 559 periarticular routes for therapeutic purposes. Another possibility would be to verify if all glucocorticoids produce systemic effect after IA or periarticular administrations, and allow the IA and periarticular use for therapeutic purposes of only the compounds with low systemic effect.

As a second step, the criteria of discrimination should be based on administration studies of the drugs using allowed and forbidden doses, and/or allowed and forbidden routes of administration, in a significant population of volunteers including individuals of different sexes and different ethnic groups. Metabolic studies of the compounds to detect as much as metabolites as possible and evaluation of all the metabolites after all these studies are recommended to look for the best marker of discrimination. Up to now, these studies have been performed for some of the compounds before establishing the threshold concentration or the reporting level, but not for all of them. If the threshold concentration or the reporting level is not based on this kind of data, it is not possible to guarantee that taking the drugs for therapeutic reasons using the allowed administration regime would not result in AAF. In this regards, a discrimination criteria needs to be developed for salmeterol. Regarding glucocorticoids, as indicated in previous paragraphs it seems almost impossible to maintain a general criterion for all the compounds taking into consideration the wide number of compounds and the diversity of administration routes, the differences in the metabolic patterns and the 
578 different pharmacological activity and therapeutic doses. For that reason, additional 579 research is needed to define the best marker for each compound.

580 Once the marker is defined, the laboratories have to develop robust methods to detect 581 the compound in routine work, and to quantify it, if necessary. Regarding the markers 582 defined up to now, common trends in the analytical methods developed in the last 583 years have been observed. Most of the markers are analyzed nowadays using LC$584 \mathrm{MS} / \mathrm{MS}$ methodology. When the threshold concentration or the reporting level does not 585 compromise the sensibility of the method, direct analysis of the diluted urine sample is 586 performed. In the cases where high sensitivity is required, a sample preparation 587 procedure is used to pre-concentrate the urine and to eliminate some of the urinary 588 interferences. One of the drawbacks of LC-MS/MS is the matrix effect that has been 589 shown to be important for some of the compounds. Total prevention of the matrix effect 590 in the initial testing procedure is complicated because general extraction protocols are 591 used to detect a broad range of substances and short chromatograms are preferable 592 because of the need of high throughput analysis. However, confirmation procedures 593 have to be designed to control that effect using more specific sample preparation 594 procedures, slow chromatographic analysis and, mainly, using isotopically labeled 595 analogues of the analytes as internal standards to correct for the matrix effect in 596 quantitative analysis.

597

598

599

600

601 


\section{Executive summary}

Some of the drugs forbidden in sports are allowed for therapeutic use under certain administration regimes. Therefore, criteria to discriminate between therapeutic use and abuse are needed.

The main groups of substances in that situation are beta-2-agonists, ephedrines and glucocorticoids.

\section{Criteria of discrimination:}

The discrimination is performed using threshold concentrations or reporting levels, and the detection of the substances in a sample is only reported as an adverse analytical finding when the concentration exceeds the threshold or the reporting level.

The criteria currently used for some of the drugs have demonstrated to present some limitations, and research for more effective criteria is needed.

An efficient discrimination needs a clear definition of the administration regime allowed, that has to be consistent from a pharmacodynamics point of view.

The criteria of discrimination should be based on pharmacokinetic studies with evaluation of the urinary concentrations of the drug or its metabolites after administration of the allowed administration regime in a representative population of volunteers. Such data is not currently available for all the compounds.

\section{Analytical methods}

Nowadays, most of the markers are measured using LC-MS/MS methods.

Direct analysis of the diluted urine samples is used when the sensibility is not compromised. If high sensitivity is required, a sample preparation procedure is used to pre-concentrate the urine and to eliminate interferences. Matrix effect needs to be controlled. 
640 Adverse analytical finding: A report from a WADA-accredited laboratory that 641 identifies in a sample the presence of a prohibited substance or its metabolites or 642 markers, or evidence of the use of a prohibited method, according to the WADA 643 requirements.

644 Threshold substance: An exogenous or endogenous prohibited substance,

645 metabolite or marker of a prohibited substance which is analyzed quantitatively and for 646 which an analytical result in excess of a pre-determined decision limit constitutes an 647 adverse analytical finding.

648

649

\section{Acknowledgements}

651 The financial support received from Consell Català de l'Esport and DIUE (2014 652 SGR 692) (Generalitat de Catalunya, Spain) and the World Antidoping Agency 653 (grants 13D22RV and 14D06RV) is acknowledged. 
1. World Anti-Doping Agency. The World Anti-Doping Code. The Prohibited List. https://www.wada-ama.org/en/resources/science-medicine/prohibitedlist (accessed May 26, 2015).

2. World Anti-Doping Agency. WADA Technical Document - TD2014DL.Decision limits for the confirmatory quantification of threshold substances. https://www.wada-ama.org/en/resources/science-medicine/td2014-dl (accessed June 03, 2015).

3. World Anti-Doping Agency. WADA Technical Document - TD2014MRPL. Minimum required performance levels for detection and identification of non-threshold substances. https://wada-mainprod.s3.amazonaws.com/resources/files/WADA-TD2014MRPL-v1Minimum-Required-Performance-Levels-EN.pdf (accessed May 26, 2015).

4. Pluim BM, de HO, Staal JB et al: beta(2)-Agonists and physical performance: a systematic review and meta-analysis of randomized controlled trials. Sports Med. 41(1), 39-57 (2011).

5. Anderson SD, Sue-Chu M, Perry CP et al: Bronchial challenges in athletes applying to inhale a beta2-agonist at the 2004 Summer Olympics. J.Allergy Clin.Immunol. 117(4), 767-773 (2006).

6. Fitch KD: beta2-Agonists at the Olympic Games. Clin.Rev.Allergy Immunol. 31(2-3), 259-268 (2006).

7. Ventura R, Segura J, Berges $R$ et al: Distinction of inhaled and oral salbutamol by urine analysis using conventional screening procedures for doping control. Ther.Drug Monit. 22(3), 277-282 (2000).

8. Eibye K, Elers J, Pedersen L et al: Formoterol concentrations in blood and urine: the World Anti-Doping Agency 2012 regulations. Med.Sci.Sports Exerc. 45(1), 16-22 (2013).

9. Ventura R, Damasceno LM, Ramirez R, Farre M, Berges R, Segura J: Evaluation of the urinary threshold concentration of formoterol in sports drug testing. Drug Test.Anal. 5(4), 266-269 (2013).

10. Pichon A, Venisse N, Krupka E, Perault-Pochat MC, Denjean A: Urinary and blood concentrations of beta2-agonists in trained subjects: comparison between routes of use. Int.J.Sports Med. 27(3), 187-192 (2006).

11. Schweizer C, Saugy M, Kamber M: Doping test reveals high concentrations of salbutamol in a Swiss track and field athlete. Clin.J.Sport Med. 14(5), 312-315 (2004).

12. Elers J, Pedersen L, Henninge J, Hemmersbach P, Dalhoff K, Backer V: The pharmacokinetic profile of inhaled and oral salbutamol in elite athletes with asthma and nonasthmatic subjects. Clin.J.Sport Med. 22(2), 140145 (2012). 
13. Sporer BC, Sheel AW, McKenzie DC: Dose response of inhaled salbutamol on exercise performance and urine concentrations. Med.Sci.Sports Exerc. 40(1), 149-157 (2008).

14. Hostrup M, Kalsen A, Auchenberg M et al: Urine concentrations of oral salbutamol in samples collected after intense exercise in endurance athletes. Drug Test.Anal. 6(6), 528-532 (2014).

15. Damasceno L, Ventura R, Ortuno J, Segura J: Derivatization procedures for the detection of beta(2)-agonists by gas chromatographic/mass spectrometric analysis. J.Mass Spectrom. 35(11), 1285-1294 (2000).

16. Ventura R, Damasceno L, Farre M, Cardoso J, Segura J: Analytical methodology for the detection of beta(2)-agonists in urine by gas chromatography-mass spectrometry for application in doping control. Analytica Chimica Acta 418(1), 79-92 (2000).

17. Damasceno L, Ventura R, Cardoso J, Segura J: Diagnostic evidence for the presence of beta-agonists using two consecutive derivatization procedures and gas chromatography-mass spectrometric analysis. J.Chromatogr.B Analyt.Technol.Biomed.Life Sci. 780(1), 61-71 (2002).

18. Zhang J, Xu Y, Di X, Wu M: Quantitation of salbutamol in human urine by liquid chromatography-electrospray ionization mass spectrometry. J.Chromatogr.B Analyt.Technol.Biomed.Life Sci. 831(1-2), 328-332 (2006).

19. Ventura R, Ramirez R, Monfort N, Segura J: Ultraperformance liquid chromatography tandem mass spectrometric method for direct quantification of salbutamol in urine samples in doping control. J.Pharm.Biomed.Anal. 50(5), 886-890 (2009).

20. Lee KM, Kim HJ, Jeong ES et al: Simple and accurate quantitative analysis of seven prohibited threshold substances in human urine by liquid chromatography/tandem mass spectrometry in doping control. Rapid Commun.Mass Spectrom. 25(16), 2261-2267 (2011).

21. Mareck U, Guddat S, Schwenke A et al: Determination of salbutamol and salbutamol glucuronide in human urine by means of liquid chromatography-tandem mass spectrometry. Drug Test.Anal. 3(11-12), 820-827 (2011).

22. Thomas A, Geyer H, Schanzer W et al: Sensitive determination of prohibited drugs in dried blood spots (DBS) for doping controls by means of a benchtop quadrupole/Orbitrap mass spectrometer. Anal.Bioanal.Chem. 403(5), 1279-1289 (2012).

23. Kiss A, Lucio M, Fildier A, Buisson C, Schmitt-Kopplin P, Cren-Olive C: Doping control using high and ultra-high resolution mass spectrometry based non-targeted metabolomics-a case study of salbutamol and budesonide abuse. PLoS.One. 8(9), e74584- (2013).

24. Berges R, Segura J, Ventura $R$ et al: Discrimination of prohibited oral use of salbutamol from authorized inhaled asthma treatment. Clin.Chem. 46(9), 1365-1375 (2000). 
25. Berges R, Segura J, de la Torre X, Ventura R: Analytical methodology for enantiomers of salbutamol in human urine for application in doping control. J.Chromatogr.B Biomed.Sci.Appl. 723(1-2), 173-184 (1999).

26. Deventer K, Pozo OJ, Delbeke FT, Van Eenoo P: Quantitative detection of inhaled formoterol in human urine and relevance to doping control analysis. Drug Test.Anal. 4(6), 449-454 (2012).

27. Mazzarino M, de la Torre X, Fiacco I, Pompei C, Calabrese F, Botre F: A simplified procedure for the analysis of formoterol in human urine by liquid chromatography-electrospray tandem mass spectrometry: application to the characterization of the metabolic profile and stability of formoterol in urine. J.Chromatogr.B Analyt.Technol.Biomed.Life Sci. 931, 75-83 (2013).

28. Lee KM, Kim HJ, Son J, Park JH, Kwon OS, Lee J: Simple quantitation of formoterol and 11-nor-Delta(9)-tetrahydrocannabinol-9-carboxylic acid in human urine by liquid chromatography-tandem mass spectrometry in doping control. J.Chromatogr.B Analyt.Technol.Biomed.Life Sci. 967, 812 (2014).

29. He G, Lu J, Wang $X$ et al: An improved liquid chromatography-tandem mass spectrometric method to quantify formoterol in human urine. J.Chromatogr.Sci. 52(8), 848-851 (2014).

30. Deventer K, Pozo OJ, Delbeke FT, Van Eenoo P: Quantitative detection of inhaled salmeterol in human urine and relevance to doping control analysis. Ther.Drug Monit. 33(5), 627-631 (2011).

31. Van Thuyne W, Van Eenoo P, Delbeke FT: Nutritional supplements: prevalence of use and contamination with doping agents. Nutr.Res.Rev. 19(1), 147158 (2006).

32. Geyer H, Parr MK, Koehler K, Mareck U, Schanzer W, Thevis M: Nutritional supplements cross-contaminated and faked with doping substances. J.Mass Spectrom. 43(7), 892-902 (2008).

33. Chan $\mathrm{KH}$, Pan RN, Hsu MC, Hsu KF: Urinary elimination of ephedrines following administration of the Traditional Chinese Medicine preparation Kakkon-to. J.Anal.Toxicol. 32(9), 763-767 (2008).

34. Chan $\mathrm{KH}$, Hsu MC, Chen FA, Hsu KF: Elimination of ephedrines in urine following administration of a Sho-seiryu-to preparation. J.Anal.Toxicol. 33(3), 162-166 (2009).

35. Deventer K, Van Eenoo P., Baele G, Pozo OJ, Van Thuyne W., Delbeke FT: Interpretation of urinary concentrations of pseudoephedrine and its metabolite cathine in relation to doping control. Drug Test.Anal. 1(5), 209-213 (2009).

36. Barroso O, Goudreault D, Carbo Banus ML et al: Determination of urinary concentrations of pseudoephedrine and cathine after therapeutic administration of pseudoephedrine-containing medications to healthy subjects: implications for doping control analysis of these stimulants banned in sport. Drug Test.Anal. 4(5), 320-329 (2012). 
37. Chester N, Mottram DR, Reilly T, Powell M: Elimination of ephedrines in urine following multiple dosing: the consequences for athletes, in relation to doping control. Br.J.Clin.Pharmacol. 57(1), 62-67 (2004).

38. Strano-Rossi S, Leone D, de la Torre X, Botre F: The relevance of the urinary concentration of ephedrines in anti-doping analysis: determination of pseudoephedrine, cathine, and ephedrine after administration of overthe-counter medicaments. Ther.Drug Monit. 31(4), 520-526 (2009).

39. Delbeke FT, Debackere M: The influence of diuretics on the excretion and metabolism of doping agents: Part VI. Pseudoephedrine. Biopharm.Drug Dispos. 12(1), 37-48 (1991).

40. Van Eenoo P, Delbeke FT, Roels K, De Backer P: Simultaneous quantitation of ephedrines in urine by gas chromatography-nitrogen-phosphorus detection for doping control purposes. J.Chromatogr.B Biomed.Sci.Appl. 760(2), 255-261 (2001).

41. Jimenez C, Ventura R, Williams J, Segura J, de la Torre R: Reference materials for analytical toxicology including doping control: freeze-dried urine samples. Analyst 129(5), 449-455 (2004).

42. Jimenez C, de la Torre R, Ventura M, Segura J, Ventura R: Stability studies of amphetamine and ephedrine derivatives in urine. J.Chromatogr. $B$ Analyt.Technol.Biomed.Life Sci. 843(1), 84-93 (2006).

43. van der Merwe PJ, Brown LW, Hendrikz SE: Simultaneous quantification of ephedrines in urine by high-performance liquid chromatography. J.Chromatogr.B Biomed.Appl. 661(2), 357-361 (1994).

44. Gmeiner G, Geisendorfer T, Kainzbauer J, Nikolajevic M, Tausch H: Quantification of ephedrines in urine by column-switching highperformance liquid chromatography. J.Chromatogr.B Analyt.Technol.Biomed.Life Sci. 768(2), 215-221 (2002).

45. Spyridaki MH, Tsitsimpikou CJ, Siskos PA, Georgakopoulos CG: Determination of ephedrines in urine by gas chromatography-mass spectrometry. J.Chromatogr.B Biomed.Sci.Appl. 758(2), 311-314 (2001).

46. Spyridaki $\mathrm{MH}$, Kiousi $\mathrm{P}$, Vonaparti $\mathrm{A}$ et al: Doping control analysis in human urine by liquid chromatography-electrospray ionization ion trap mass spectrometry for the Olympic Games Athens 2004: determination of corticosteroids and quantification of ephedrines, salbutamol and morphine. Anal.Chim.Acta 573-574, 242-249 (2006).

47. Deventer K, Pozo OJ, Van Eenoo P, Delbeke FT: Development and validation of an LC-MS/MS method for the quantification of ephedrines in urine. J.Chromatogr.B Analyt.Technol.Biomed.Life Sci. 877(4), 369-374 (2009).

48. Badoud F, Grata E, Perrenoud L, Saugy M, Rudaz S, Veuthey JL: Fast analysis of doping agents in urine by ultra-high-pressure liquid chromatographyquadrupole time-of-flight mass spectrometry. II: Confirmatory analysis. J.Chromatogr.A 1217(25), 4109-4119 (2010). 
49. Gray N, Musenga A, Cowan DA, Plumb R, Smith NW: A simple high pH liquid chromatography-tandem mass spectrometry method for basic compounds: application to ephedrines in doping control analysis. J.Chromatogr.A 1218(15), 2098-2105 (2011).

50. Heaton J, Gray N, Cowan DA, Plumb RS, Legido-Quigley C, Smith NW: Comparison of reversed-phase and hydrophilic interaction liquid chromatography for the separation of ephedrines. J.Chromatogr.A 1228, 329-337 (2012).

51. Gray N, Heaton J, Musenga A, Cowan DA, Plumb RS, Smith NW: Comparison of reversed-phase and hydrophilic interaction liquid chromatography for the quantification of ephedrines using medium-resolution accurate mass spectrometry. J.Chromatogr.A 1289, 37-46 (2013).

52. Monfort N, Martinez L, Berges R, Segura J, Ventura R: Screening method for stimulants in urine by UHPLC-MS/MS: identification of isomeric compounds. Drug Test.Anal. (2015).

53. King JH, Weimer JR: Prednisone (meticorten) and prednisolone (meticortelone) in ophthamology; experimental and clinical studies. AMA.Arch.Ophthalmol. 54(1), 46-54 (1955).

54. Duclos M: Evidence on ergogenic action of glucocorticoids as a doping agent risk. Phys.Sportsmed. 38(3), 121-127 (2010).

55. Simoes SMS, Calçada M, Horta L, de la Torre X. Methylprednisolone detection in urine following local and oral administrations. In: Recent Advances in Doping Analysis (13). Schänzer W, Geyer H, Gotzmann A, Mareck U (Eds.). 411-414 (2005)

56. Mazzarino M, Bragano MC, Garribba F, Rossi F, Botre F. The idea of a threshold value for synthetic glucocorticoids: urinary excretion studies following different routes of administration. In: Recent Advances in Doping Analysis (13). Schänzer W, Geyer H, Gotzmann A, Mareck U (Eds.). 465-468 (2005)

57. Bredehöft M, Thevis M, Schänzer W. Excretion study: betamethasone-17,21propionate ointment and possible metabolites in man analyzed with LCESI-MS/MS. In: Recent Advances in Doping Analysis (13). Schänzer W, Geyer H, Gotzmann A, Mareck U (Eds.). 415-418 (2005)

58. Deventer K, Mikulcikova P, Van Hoecke H, Van Eenoo P, Delbeke FT: Detection of budesonide in human urine after inhalation by liquid chromatography-mass spectrometry. J.Pharm.Biomed.Anal. 42(4), 474479 (2006).

59. Panusa A, Regazzoni L, Aldini G et al: Urinary profile of methylprednisolone acetate metabolites in patients following intra-articular and intramuscular administration. Anal.Bioanal.Chem. 400(1), 255-267 (2011).

60. Matabosch X, Pozo OJ, Pérez-Mañá C et al: Identification of budesonide metabolites in human urine after oral administration. Anal.Bioanal.Chem. 404(2), 325-340 (2012). 
61. Pozo OJ, Marcos J, Matabosch X, Ventura R, Segura J: Using complementary mass spectrometric approaches for the determination of methylprednisolone metabolites in human urine. Rapid Commun.Mass Spectrom. 26(5), 541-553 (2012).

62. Matabosch X, Pozo OJ, Pérez-Mañá $C$ et al: Discrimination of prohibited oral use from authorized inhaled treatment of budesonide in sports. Ther.Drug Monit. 35(1), 118-128 (2013).

63. Matabosch X, Pozo OJ, Monfort $\mathrm{N}$ et al: Urinary profile of methylprednisolone metabolites after oral and topical administrations. J.Steroid Biochem.Mol.Biol. 138(C), 214-221 (2013).

64. Matabosch X, Pozo OJ, Papaseit.E. et al: Detection and characterization of triamcinolone acetonide metabolites in human urine by LC-MS/MS after intramuscular administration. Rapid Commun.Mass Spectrom. 28(16), 1829-1839 (2014).

65. Matabosch X, Monfort N, Pozo O et al. Urinary profile of betamethasone metabolites after different administration routes. Presented at: $32 n d$ Cologne Workshop on Dope Analysis. Cologne, Germany, 30th March 4 April 2014.

66. Matabosch X, Pozo OJ, Monfort $\mathrm{N}$ et al: Detection and characterization of betamethasone metabolites in human urine by LC-MS/MS. Drug Test.Anal. 7(8), 663-672 (2015).

67. Matabosch X, Pozo OJ, Pérez-Mañá $C$ et al: Evaluation of the reporting level to detect triamcinolone acetonide misuse in sports. J.Steroid Biochem.Mol.Biol. 145, 94-102 (2015).

68. Matabosch X, Pozo OJ, Perez-Mana C, Papaseit E, Segura J, Ventura R: Detection and characterization of prednisolone metabolites in human urine by LC-MS/MS. J.Mass Spectrom. 50(3), 633-642 (2015).

69. Chang CW, Huang TY, Tseng YC, Chang-Chien GP, Lin SF, Hsu MC: Positive doping results caused by the single-dose local injection of triamcinolone acetonide. Forensic Sci.Int. 244, 1-6 (2014).

70. World Anti-Doping Agency. 2013 Anti-Doping Testing Figures. https://www.wada-ama.org/en/resources/laboratories/2013-anti-dopingtesting-figures-laboratory-report (accessed May 26, 2015).

71. Deventer K, Delbeke FT: Validation of a screening method for corticosteroids in doping analysis by liquid chromatography/tandem mass spectrometry. Rapid Commun.Mass Spectrom. 17(18), 2107-2114 (2003).

72. Mazzarino M, Turi S, Botre F: A screening method for the detection of synthetic glucocorticosteroids in human urine by liquid chromatography-mass spectrometry based on class-characteristic fragmentation pathways. Anal.Bioanal.Chem. 390(5), 1389-1402 (2008).

73. Gless KH, Klee HR, Vecsei P, Weber M, Haack D, Lichtwald K: [Plasma concentration and systemic effect of betamethasone after intra-articular 

(1981).

74. Derendorf H, Mollmann H, Gruner A, Haack D, Gyselby G: Pharmacokinetics and pharmacodynamics of glucocorticoid suspensions after intraarticular administration. Clin.Pharmacol.Ther. 39(3), 313-317 (1986).

75. Mazzarino M, Rossi F, Giacomelli L, Botre F: Effect of the systemic versus inhalatory administration of synthetic glucocorticoids on the urinary steroid profile as studied by gas chromatography-mass spectrometry. Analytica Chimica Acta 559(1), 30-36 (2006).

76. Fluri K, Rivier L, Dienes-Nagy A et al: Method for confirmation of synthetic corticosteroids in doping urine samples by liquid chromatographyelectrospray ionisation mass spectrometry. J.Chromatogr.A 926(1), 8795 (2001).

77. Segura J, Ventura R, Jurado C: Derivatization procedures for gas chromatographic-mass spectrometric determination of xenobiotics in biological samples, with special attention to drugs of abuse and doping agents. J.Chromatogr.B Biomed.Sci.Appl. 713(1), 61-90 (1998).

78. Pozo OJ, Ventura R, Monfort N, Segura J, Delbeke FT: Evaluation of different scan methods for the urinary detection of corticosteroid metabolites by liquid chromatography tandem mass spectrometry. J.Mass Spectrom. 44(6), 929-944 (2009).

79. Marcos J, Renau N, Casals G, Segura J, Ventura R, Pozo OJ: Investigation of endogenous corticosteroids profiles in human urine based on liquid chromatography tandem mass spectrometry. Anal.Chim.Acta 812, 92104 (2014).

80. Tudela E, Munoz G, Munoz-Guerra JA: Matrix effect marker for multianalyte analysis by LC-MS/MS in biological samples. J.Chromatogr. $B$ Analyt.Technol.Biomed.Life Sci. 901, 98-106 (2012).

81. Savel'eva NB, Bykovskaia NI, Dikunets MA, Bolotov SL, Rodchenkov GM: [The use of the deuterated internal standard for morphine quantitation for the purpose of doping control by gas chromatography with mass-selective detection]. Sud.Med.Ekspert. 53(3), 29-32 (2010).

82. Solans A, de la Torre R, Segura J: Determination of morphine and codeine in urine by gas chromatography-mass spectrometry. J.Pharm.Biomed.Anal. 8(8-12), 905-909 (1990).

83. Van Thuyne W, Van Eenoo P, Delbeke FT: Urinary concentrations of morphine after the administration of herbal teas containing Papaveris fructus in relation to doping analysis. J.Chromatogr.B Analyt.Technol.Biomed.Life Sci. 785(2), 245-251 (2003).

84. Gorgens C, Guddat S, Orlovius AK et al: "Dilute-and-inject" multi-target screening assay for highly polar doping agents using hydrophilic interaction liquid chromatography high resolution/high accuracy mass spectrometry for sports drug testing. Anal.Bioanal.Chem. (2015). 
958

959

960

961 
Table 1. Threshold concentrations and decision limits for substances in anti-doping 963 control. Modification of Table 1 in the WADA Technical Document TD2014DL [2].

\begin{tabular}{|l|c|c|c|c|}
\hline Substance & Units & Threshold & $\begin{array}{c}\text { Uncertainty } \\
(\%)^{a}\end{array}$ & Decision limit \\
\hline 19-Norandrosterone & $\mathrm{ng} / \mathrm{ml}$ & 2.0 & 15 & 2.5 \\
\hline THC metabolite ${ }^{\mathrm{b}}$ & $\mathrm{ng} / \mathrm{ml}$ & 150 & 10 & 180 \\
\hline Salbutamol & $\mu \mathrm{g} / \mathrm{ml}$ & 1.0 & 10 & 1.2 \\
\hline Formoterol & $\mathrm{ng} / \mathrm{ml}$ & 40 & 15 & 50 \\
\hline Glycerol & $\mathrm{mg} / \mathrm{ml}$ & 4.3 & 15 & 5.3 \\
\hline Morphine & $\mu \mathrm{g} / \mathrm{ml}$ & 1.0 & 15 & 1.3 \\
\hline Cathine & $\mu \mathrm{g} / \mathrm{ml}$ & 5.0 & 10 & 6.0 \\
\hline Ephedrine & $\mu \mathrm{g} / \mathrm{ml}$ & 10 & 5 & 11 \\
\hline Methylephedrine & $\mu \mathrm{g} / \mathrm{ml}$ & 10 & 5 & 11 \\
\hline Pseudoephedrine & $\mu \mathrm{g} / \mathrm{ml}$ & 150 & 5 & 170 \\
\hline
\end{tabular}

${ }^{a}$ Maximum combined relative standard uncertainty

b11-nor- $\Delta$ 9-tetrahydrocannabinol-9-carboxylic acid 
968 Figure 1. Quantitation of formoterol by LC-MS/MS: Chromatograms obtained after 969 analysis of a blank urine (A), a sample containing $68.7 \mathrm{ng} / \mathrm{ml}$ of formoterol (B), and a 970 calibration sample containing $50 \mathrm{ng} / \mathrm{ml}$ of formoterol (C). Ion transitions monitored: $971351>155$ for formoterol-d6, used as internal standard; and 345>327 and 345>149 for 972 formoterol.

973

(A)
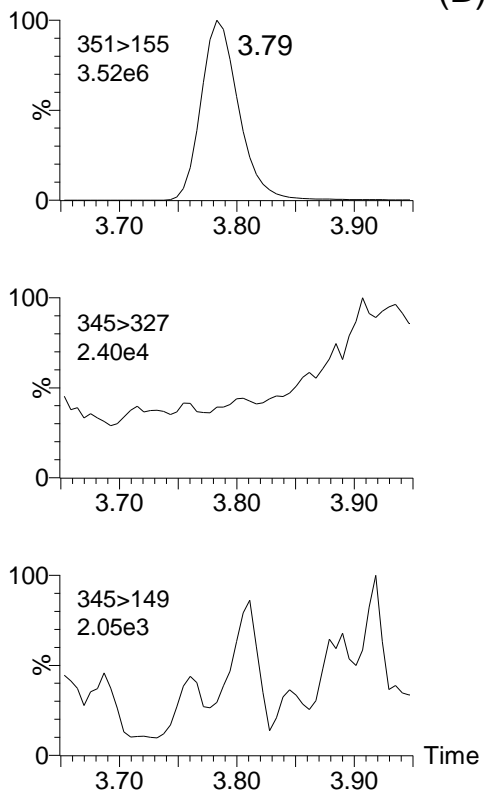

(B)
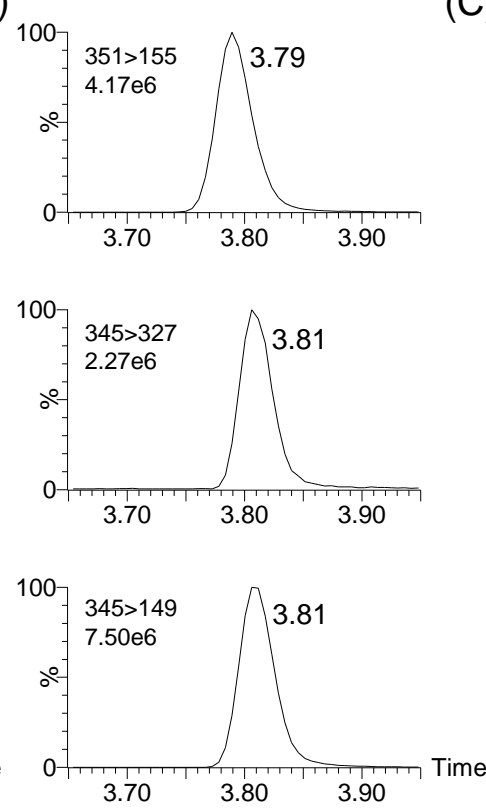

(C)
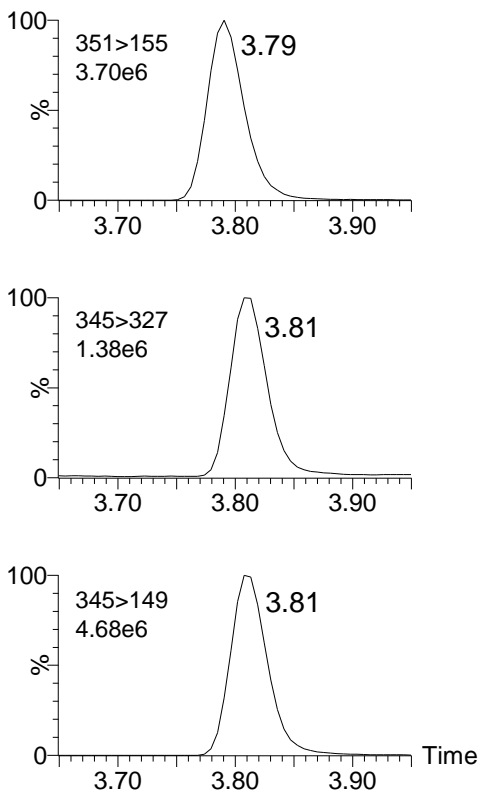
976 Figure 2. Separation of ephedrines using LC-MS/MS analysis.

977
(A) ${ }_{100} \mathrm{~A} 1{ }^{\mathrm{A} 2}$

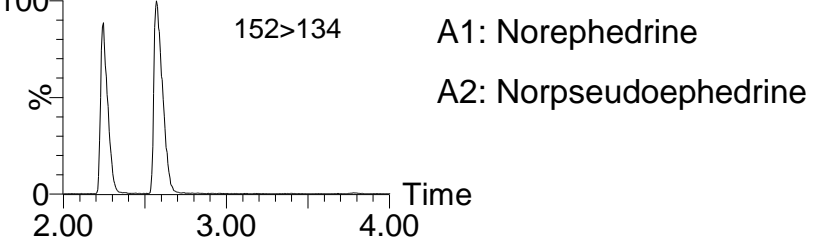
(B)
B1 B2

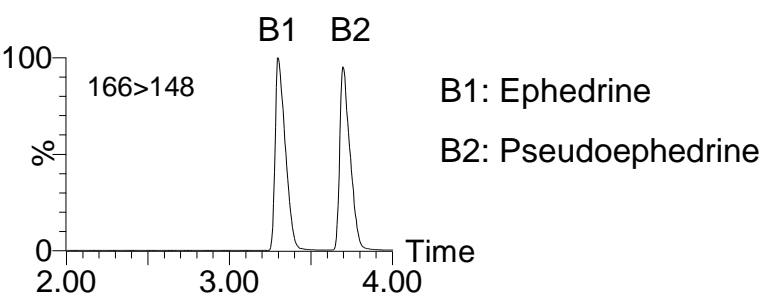


Figure 3. Prednisolone metabolites detected after oral administration by LC-MS/MS.

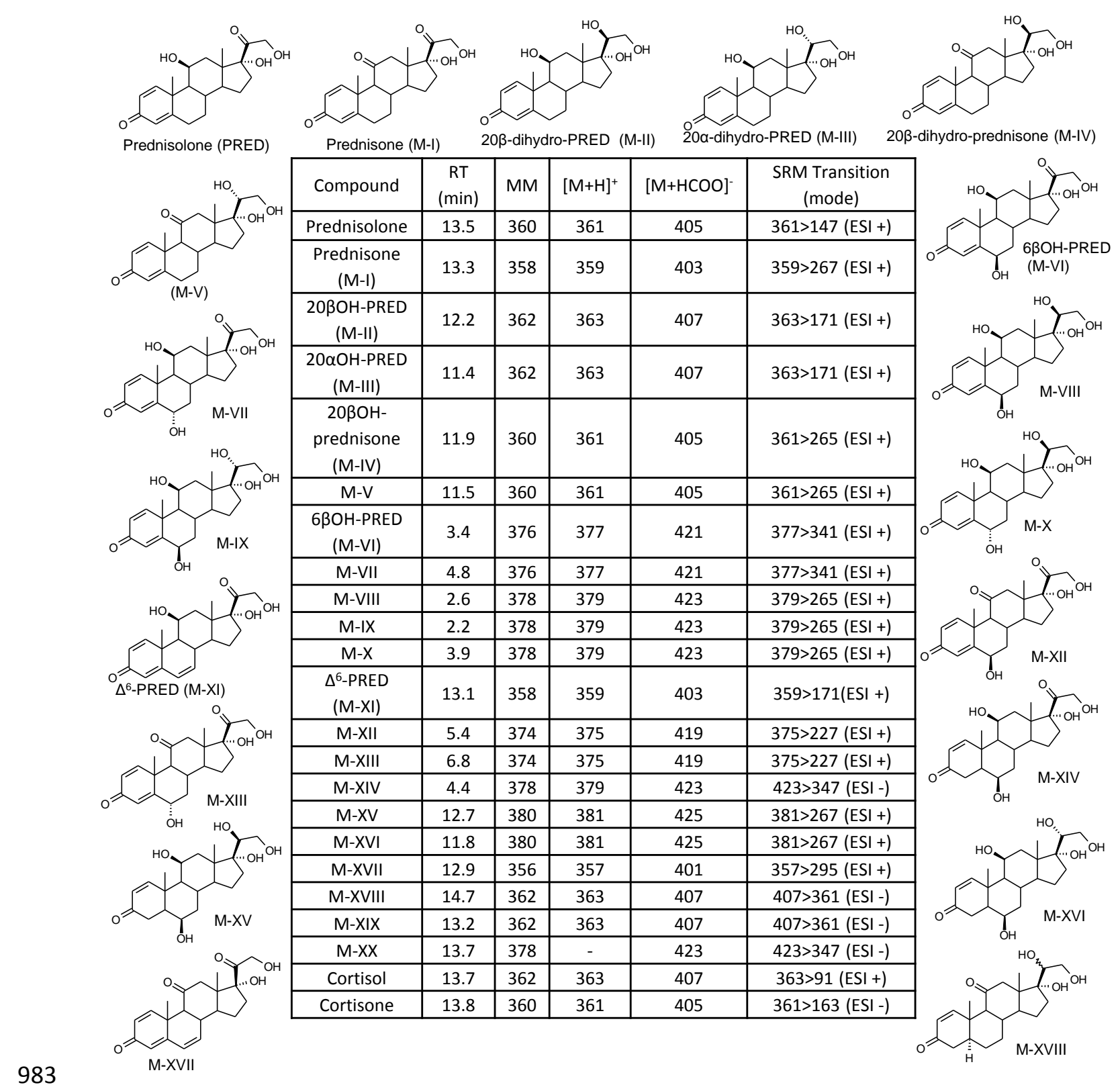


Figure 4. LC-MS/MS analysis of budesonide and metabolites: Chromatograms of budesonide and its main metabolites after inhaled and oral administrations of budesonide. For all compounds, chromatograms are Y-scaled at the highest peak of the sample collected $0-4 \mathrm{~h}$ after oral administration.

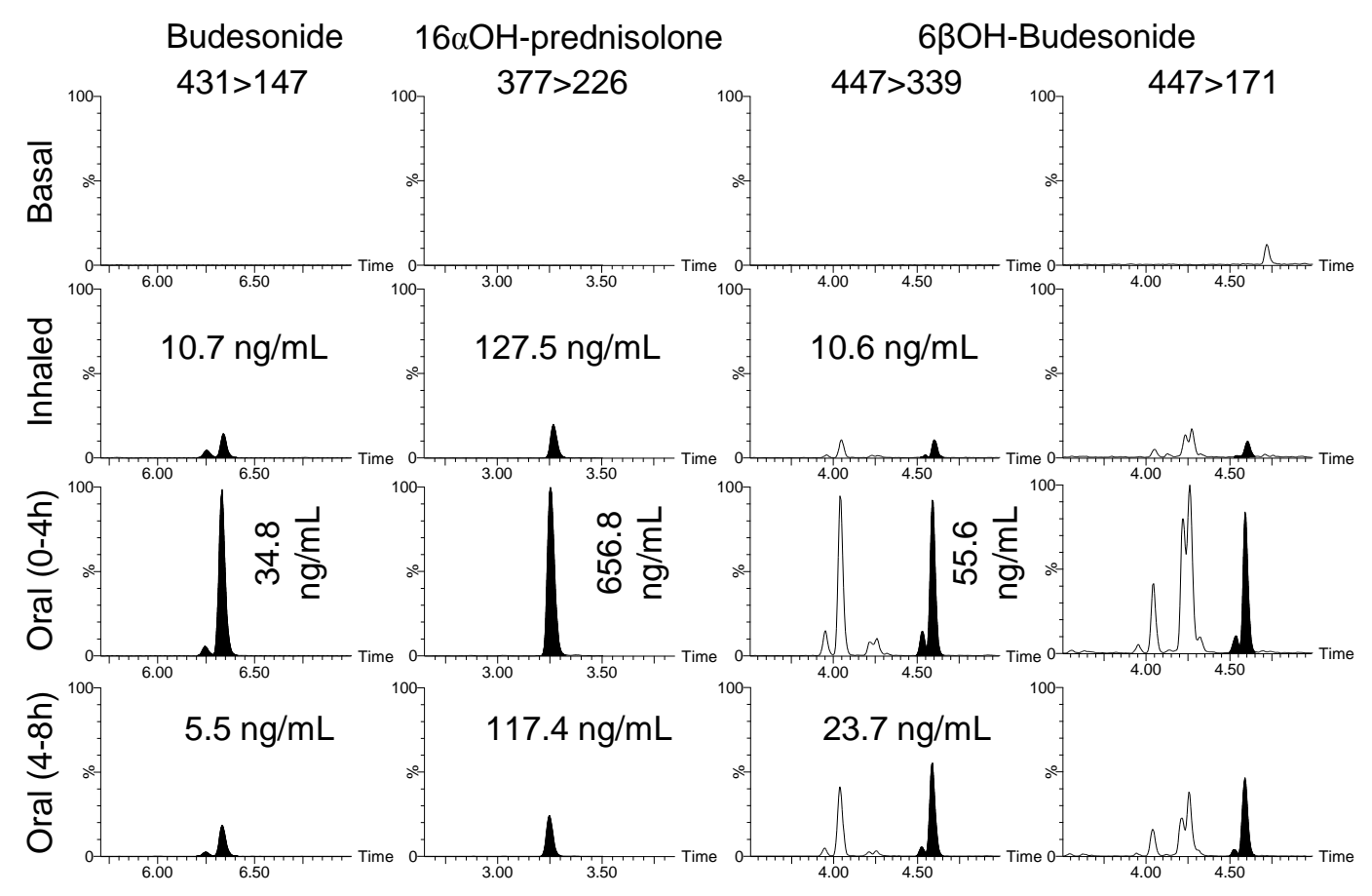

991 